\title{
Colossal thermomagnetic response in the exotic superconductor $\mathrm{URu}_{2} \mathrm{Si}_{2}$
}

\author{
T. Yamashita', Y. Shimoyama ${ }^{1}$, Y. Haga ${ }^{2}$, T. D. Matsuda ${ }^{3}$, E. Yamamoto ${ }^{2}$, Y. Onuki ${ }^{2,4}$, H. Sumiyoshi', \\ S. Fujimoto ${ }^{5}$, A. Levchenko ${ }^{6}$, T. Shibauchi ${ }^{1,7}$ and Y. Matsuda ${ }^{1 \star}$
}

The superconducting fluctuation effect, due to preformed Cooper pairs above the critical temperature $T_{c}$, has been generally understood by the standard Gaussian fluctuation theories in most superconductors ${ }^{1}$. The transverse thermoelectric (Nernst) effect is particularly sensitive to the fluctuations, and the large Nernst signal found in the pseudogap regime of the underdoped cuprates ${ }^{2,3}$ has raised much debate. Here we report on the observation of a colossal Nernst signal due to the superconducting fluctuations in the heavy-fermion superconductor $\mathrm{URu}_{2} \mathrm{Si}_{2}$. The Nernst coefficient is anomalously enhanced (by a factor of $\sim 10^{6}$ ) as compared with the theoretically expected value of the Gaussian fluctuations. Moreover, contrary to the conventional wisdom, the enhancement is more significant with a reduction of the impurity scattering rate. This unconventional Nernst effect intimately reflects the highly unusual superconducting state of $\mathrm{URu}_{2} \mathrm{Si}_{2}$. The results invoke possible chiral or Berry-phase fluctuations associated with the broken time-reversal symmetry ${ }^{4-7}$ of the superconducting order parameter.

Measurements of the Nernst effect provide a unique opportunity to study the superconducting fluctuations deep inside the normal state above $T_{c}$ (refs $2,3,8-12$ ). The Nernst signal $N$ is the electric field $E_{y}(\| y)$ response to a transverse temperature gradient $\nabla_{x} T(\| x)$ in the presence of a magnetic field $H(\| z)$, and is given by $N \equiv E_{y} /\left(-\nabla_{x} T\right)$. The Nernst coefficient, defined as $v \equiv N / \mu_{0} H$ above $T_{c}$, consists of two contributions generated by different mechanisms: $v=v^{\mathrm{s}}+v^{\mathrm{N}}$. The first term, $v^{\mathrm{s}}$, represents the contribution of superconducting fluctuations of either amplitude or phase of the order parameter, which is always positive ${ }^{13}$. The second term, $v^{\mathrm{N}}$, represents the contribution from the normal quasiparticles, which can be either positive or negative ${ }^{14}$. The second contribution is usually small in conventional metals. In almost all superconductors the superconducting fluctuation contribution to the Nernst effect can be accounted for by the Gaussian-type fluctuations ${ }^{13,15-18}$. Recently, a large Nernst signal has been reported in the pseudogap state of the hole-doped underdoped high- $T_{c}$ cuprates, which has been discussed in terms of possible vortex-like excitations of phase disordered superconductors ${ }^{2,3,8}$. Although its origin is still controversial, these results imply that the fluctuationinduced Nernst signal above $T_{c}$ is intimately related to the exotic superconducting state below $T_{c}$.

The heavy-fermion compound $\mathrm{URu}_{2} \mathrm{Si}_{2}$ exhibits unconventional superconductivity $\left(T_{\mathrm{c}}=1.45 \mathrm{~K}\right)$. This compound is distinguished from the other heavy-fermion compounds by the fact that the mysterious hidden-order transition takes place at $T_{\mathrm{HO}}=17.5 \mathrm{~K}$, and no evidence of magnetic order has been found below $T_{\mathrm{HO}}$ (ref. 19). This system has been suggested to be a candidate as a chiral $d$-wave superconductor that spontaneously breaks time-reversal symmetry (TRS) in the superconducting state $e^{4-7}$. Indeed, the angular dependence of the thermal conductivity and specific heat in magnetic fields indicate the presence of point nodes in the order parameter, and a chiral $d$-wave pairing symmetry in a complex form of $k_{z}\left(k_{x} \pm i k_{y}\right)$ has been proposed ${ }^{4,5}$. Very recently, the broken TRS has also been reported as a result of polar Kerr effect measurements (A. Kapitulnik, private communications). On the basis of these results, possible Weyl-type topological superconducting states have been discussed ${ }^{20}$. It is therefore highly intriguing to examine the superconducting fluctuations in $\mathrm{URu}_{2} \mathrm{Si}_{2}$.

Figure 1a shows $v(T)$ in the zero-field limit (see also Supplementary Fig. 2a) and Fig. $1 \mathrm{~b}$ the in-plane resistivity $\rho_{x x}$ of ultraclean $\mathrm{URu}_{2} \mathrm{Si}_{2}$ single crystals $\left(T_{\mathrm{c}}=1.45 \mathrm{~K}\right)$ with residual resistivity ratios (RRR) of $1,080(\# 1)$ and $620(\# 2)$. It has been reported that for RRR $\gtrsim 30$, both $T_{\mathrm{HO}}$ and $T_{\mathrm{c}}$ are almost independent of the RRR value ${ }^{21}$. Above $T_{\mathrm{HO}}, v(T)$ is negligibly small and exhibits a marked increase on entering the hidden-order state. Below $T^{*} \sim 5 \mathrm{~K}, v(T)$ shows a further enhancement and increases divergently on approaching $T_{\mathrm{c}}$ (Fig. 1a,b). The inset of Fig. $1 \mathrm{~b}$ shows the $T$-dependence of the ratios of Nernst coefficient and conductivity $\sigma=1 / \rho_{x x}$ in the two crystals, $r_{v} \equiv v(\# 1) / v(\# 2)$ and $r_{\sigma} \equiv \rho_{x x}(\# 2) / \rho_{x x}(\# 1)$, respectively. In contrast to the nearly $T$-independent $r_{\sigma}, r_{v}$ increases steeply below $\sim T^{*}$, suggesting the appearance of an additional mechanism that generates the enhanced Nernst effect in the cleaner crystal. These results indicate that the superconducting fluctuation effect sets in below $\sim T^{*}$. As discussed later, this is supported by the $H$-dependence of the Nernst effect. In magnetic fields, $v(T)$ vanishes just below the vortex lattice melting temperature, $T_{\text {melt }}$ (Fig. $\left.2 a\right)^{6}$.

First, we discuss the Nernst signal in the range of $T$ that is free from the superconducting fluctuations $\left(T^{*} \lesssim T \lesssim T_{\mathrm{HO}}\right)$. As shown in Fig. 2b, increasing RRR or the scattering time $\tau$ leads to an enhancement of $v^{\mathrm{N}}$. Within the Boltzmann theory, when $\tau$ is weakly dependent on energy, $v^{\mathrm{N}}$ can be expressed as $\nu^{\mathrm{N}}=\left(\pi^{2} / 3\right)\left(k_{\mathrm{B}}^{2} T / m^{*}\right)\left(\tau / \varepsilon_{\mathrm{F}}\right)($ refs 10,22$)$, where $k_{\mathrm{B}}$ is the Boltzmann constant, $m^{*}$ is the effective mass and $\varepsilon_{\mathrm{F}}$ is the Fermi energy. The striking enhancement of $v$ below $T_{\mathrm{HO}}$ is attributed to the strong reduction of $\varepsilon_{\mathrm{F}}$ associated with the disappearance of carriers and

\footnotetext{
${ }^{1}$ Department of Physics, Kyoto University, Kyoto 606-8502, Japan. ${ }^{2}$ Advanced Science Research Center, Japan Atomic Energy Agency, Tokai 319-1195, Japan. ${ }^{3}$ Department of Physics, Tokyo Metropolitan University, Hachioji, Tokyo 192-0397, Japan. ${ }^{4}$ Department of Physics and Earth Sciences, University of the Ryukyus, Nishihara, Okinawa 903-0213, Japan. ${ }^{5}$ Department of Materials Engineering Science, Osaka University, Toyonaka, Osaka 560-8531, Japan. ${ }^{6}$ Department of Physics and Astronomy, Michigan State University, East Lansing, Michigan 48824, USA. 7 Department of Advanced Materials Science, University of Tokyo, Kashiwa, Chiba 277-8561, Japan. ${ }^{\star}$--mail: matsuda@scphys.kyoto-u.ac.jp
} 


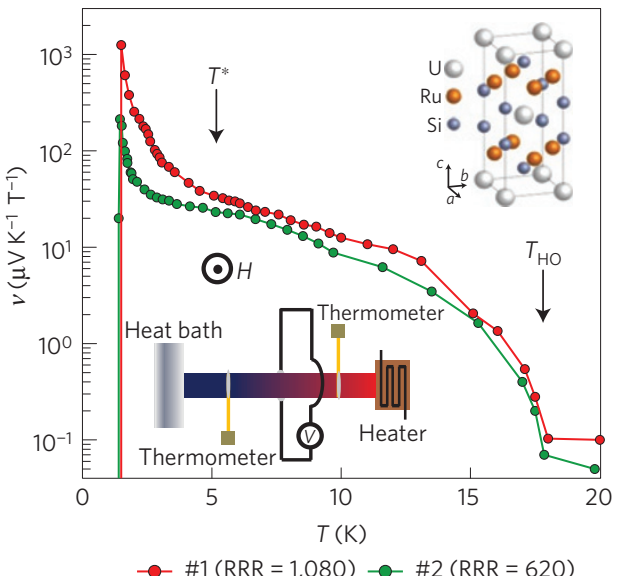

b

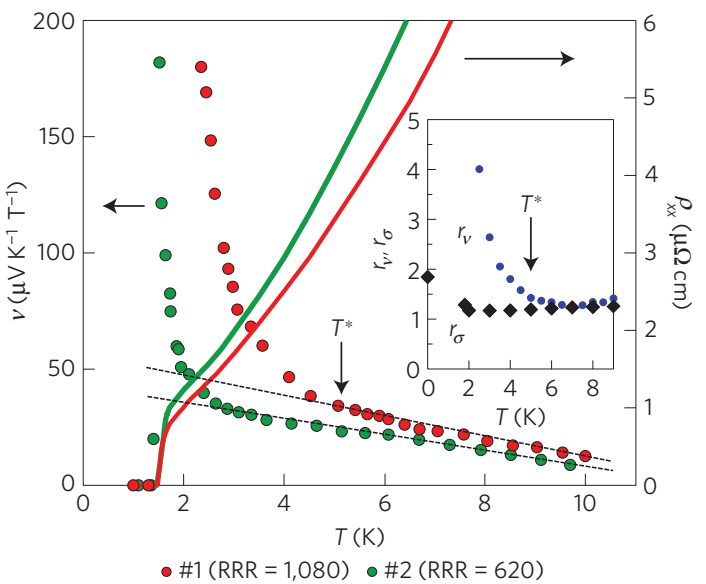

Figure 1 | Transverse thermoelectric response in $\mathrm{URu}_{2} \mathrm{Si}_{2}$.

a, $T$-dependence of the Nernst coefficient $v$ in the zero-field limit $(\mathbf{H} \| c)$ for single crystals $\# 1(R R R=1,080)$ and $\# 2(R R R=620)$. The $R R R$ values are determined from $\rho(300 \mathrm{~K}) / \rho_{0}$ by assuming the $T$-dependence of the in-plane resistivity $\rho_{x x}$ as $\rho_{x x}(T)=\rho_{0}+A T^{n}$, with $n=1.5$ and 1.7 for $\# 1$ and $\# 2$, respectively, below $6 \mathrm{~K}$. In both crystals, $T_{c}$ defined by the point of zero resistivity is $1.45 \mathrm{~K}$. The upper inset illustrates the crystal structure of $\mathrm{URu}_{2} \mathrm{Si}_{2}$ and the lower inset is a schematic of the measurement set-up. b, Low-temperature data of $v(T)$ and $\rho_{x x}(T)$ for crystals \#1 and \#2. Below $T^{*}, v$ rises sharply above the $T$-linear dependence extrapolated from higher temperatures (dashed lines). The inset shows the $T$-dependence of the ratios of the Nernst coefficient and conductivity of the two crystals, $r_{v}=v(\# 1) / v(\# 2)$ and $r_{\sigma}=\rho_{x x}(\# 2) / \rho_{X x}(\# 1)$.

concomitant enhancement of $\tau$, both of which have been reported previously ${ }^{4}$. The fact that $r_{v}$ above $T^{*}$ coincides well with $r_{\sigma}$ (inset of Fig. 1b) provides quantitative support of $v^{\mathrm{N}} \propto \tau$.

At lower temperatures below $T^{*}, v$ of clean crystals becomes huge, especially in the vicinity of $T_{\mathrm{c}}$. Indeed, $v$ of the cleanest crystal \#1 is comparable to that of pure semimetal $\mathrm{Bi}$, with the largest Nernst coefficient reported so far ${ }^{22}$. Moreover, the combination of the large Nernst signal and high conductivity in this system leads to a sizeable thermomagnetic figure of merit $Z T_{\epsilon}=N^{2} \sigma T / \kappa(\kappa$ is the thermal conductivity), which exceeds by far the values of previously studied materials (Fig. 2c) ${ }^{23,24}$.

Now we discuss the fluctuation-induced Nernst coefficient $v^{\mathrm{S}}$ The enhancement of $r_{v}(T)$ below $T^{*}$ (inset of Fig. 1b) and no discernible enhancement of $v(T)$ near $T_{c}$ for RRR $\sim 30$ (inset of Fig. 2b) indicate that $v^{\mathrm{S}}$ is greatly enhanced with $\tau$. We stress that this $\tau$-dependence of $\nu^{S}$ is opposite to that expected in the conventional Gaussian fluctuation theories, which predict $\nu^{\mathrm{S}} \propto \rho_{x x} \propto 1 / \tau$ (refs $13,16-18$ ). It has also been reported that in underdoped cuprates the introduction of impurities by irradiation enhances $v^{\mathrm{S}}$ (ref. 8), which is again opposite to the $\mathrm{URu}_{2} \mathrm{Si}_{2}$ case. It is intriguing to compare the present results with $\mathrm{CeCoIn}_{5}$, which shares several common features with $\mathrm{URu}_{2} \mathrm{Si}_{2}$, such as heavyfermion unconventional superconductivity with a nodal gap and similar $T_{\mathrm{c}}$ and upper critical fields. It should be stressed that in very pure CeCoIn ${ }_{5}$, with $\rho\left(T_{c}^{+}\right) \approx 4 \mu \Omega \mathrm{cm}$, which is of the same order as that of our $\mathrm{URu}_{2} \mathrm{Si}_{2}$ crystals, no discernible $v^{\mathrm{S}}$ is observed ${ }^{25,26} ; v^{\mathrm{S}}$ is at least two orders of magnitude smaller in pure $\mathrm{CeCOIn}_{5}$ than in $\mathrm{URu}_{2} \mathrm{Si}_{2}$. These results thus highlight an essential difference in the superconducting fluctuations between $\mathrm{URu}_{2} \mathrm{Si}_{2}$ and the other unconventional superconductors.

The unusual nature of the superconducting fluctuations in $\mathrm{URu}_{2} \mathrm{Si}_{2}$ is further revealed by the off-diagonal component of the thermoelectric tensor (Peltier coefficient) $\alpha_{x y}$, which is a more fundamental quantity associated with the fluctuations than the Nernst coefficient. The relation between $v$ and other coefficients is given as $\nu=1 / \mu_{0} H\left(\alpha_{x y} \rho_{x x}-S \tan \theta_{\mathrm{H}}\right)$, where $\mu_{0}$ is the vacuum permeability, $S$ is the Seebeck coefficient and $\theta_{\mathrm{H}}$ is the Hall angle. Throughout the $T$ - and $H$-regions in the present study, $\alpha_{x y} \rho_{x x} \gg S \tan \theta_{\mathrm{H}}$ - that is, $v \approx \alpha_{x y} \rho_{x x} / \mu_{0} H$ (Supplementary Figs 1 and $2 b$ ). Figure 3 shows the $T$-dependence of the fluctuationinduced Peltier coefficient $\alpha_{x y}^{S}$ divided by $\mu_{0} H$ in the zero-field limit. To determine $\alpha_{x y}^{S}$, the deviation from $T$-linear behaviour in $v(T)$ (dashed lines in Fig. 1b) is attributed to $v^{\mathrm{s}}$. The Peltier coefficient that results from the Gaussian (Aslamazov-Larkin) fluctuations is given by

$$
\alpha_{x y}^{\mathrm{AL}}(T)=\frac{1}{12 \pi} \frac{k_{\mathrm{B}} e}{\hbar} \frac{\xi_{a b}^{2}(T)}{\ell_{H}^{2} \xi_{c}(T)} \propto \frac{1}{\sqrt{\ln \left(T / T_{c}\right)}}
$$

where $\xi_{a b, c}(T)=\xi_{a b, c}(0) / \sqrt{\ln \left(T / T_{c}\right)}$ are the fluctuation coherence lengths parallel to the $a b$ plane and $c$ axis, and $\ell_{H}=\sqrt{\hbar / 2 e \mu_{0} H}$ is the magnetic length, with $e$ being the electronic charge and $\hbar$ Planck's constant. The blue line in Fig. 3 shows the $T$-dependence of $\alpha_{x y}^{\mathrm{AL}} / \mu_{0} H$, calculated using $\xi_{a b}(0)=10 \mathrm{~nm}$ and $\xi_{c}(0)=3.3 \mathrm{~nm}$, which are determined by the initial slope of upper critical fields at $T_{c}$. What is most spectacular is that the observed $\alpha_{x y}^{S} / \mu_{0} H$ is four to six orders of magnitude greater than $\alpha_{x y}^{\mathrm{AL}} / \mu_{0} H$ given by equation (1). Moreover, the $T$-dependence of $\alpha_{x y}^{S}(T)$ is much steeper than $\alpha_{x y}^{\mathrm{AL}}(T)$.

We stress that the observed $\alpha_{x y}^{S}(T)$ far exceeding $\alpha_{x y}^{\mathrm{AL}}(T)$ indeed originates from the superconducting fluctuations. This is evidenced by the steep enhancement of both $v(T)$ and $r_{v}(T)$ below $T^{*}$. The $H$-dependence of $\alpha_{x y}(H)$ provides quantitative support for this. It has been pointed out that the size of superconducting fluctuation is set by the coherence length at low fields, whereas it is set by the magnetic length at high fields. As a result, $\alpha_{x y}(H)$ peaks at a characteristic field $H^{*}$, where $\xi_{a b}(T)=\ell_{H}\left(H^{*}\right)$, so that $\mu_{0} H^{*}=\Phi_{0} /\left(2 \pi \xi_{a b}^{2}(0)\right) \ln \left(T / T_{\mathrm{c}}\right)$, where $\Phi_{0}$ is the flux quantum. The peak field $H^{*}$ is called the 'ghost critical field', and has been reported both in conventional and unconventional superconductors ${ }^{11,12}$. As shown in Fig. 4, at all temperatures of interest, $\alpha_{x y}(H)$ exhibits a peak. The inset of Fig. 4 shows the peak field plotted as a function of $\ln \left(T / T_{\mathrm{c}}\right)$. The solid line, which represents $H^{*}(T)$ calculated by using $\xi_{a b}(0)=10 \mathrm{~nm}$, is quantitatively consistent with the peak field.

We discuss several possible origins for the observed colossal Nernst signal. First, equation (1) assumes the diffusive limit, $k_{\mathrm{B}} T \ll \hbar / \tau$, whereas the present $\mathrm{URu}_{2} \mathrm{Si}_{2}$ seems to be in the ballistic regime, $k_{\mathrm{B}} T \gg \hbar / \tau$. Although an extension of equation (1) to the clean case (see Supplementary Information) reveals the enhancement of $\alpha_{x y}^{S}$ with $\tau$, this enhancement is slower than the reduction of $\rho_{x x}$, so that $\nu^{S} \propto \alpha_{x y}^{S} \rho_{x x}$ is still suppressed for larger $\tau$, which is inconsistent with Fig. 2 b. Second, in the multiband system, each band with different effective coherence lengths contributes differently to the total $\alpha_{x y}^{S}$. To explain the observed $\alpha_{x y}^{S}$, however, small bands with extremely large effective coherence lengths, 


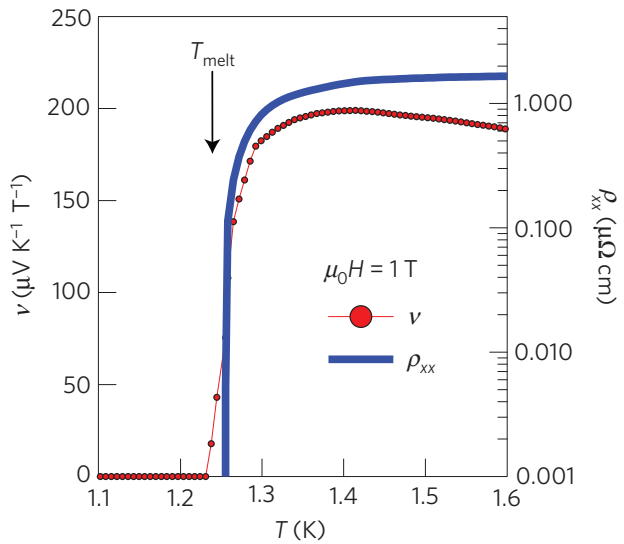

C

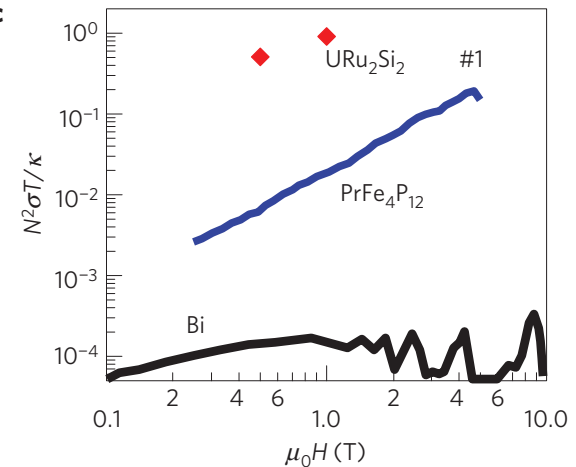

b

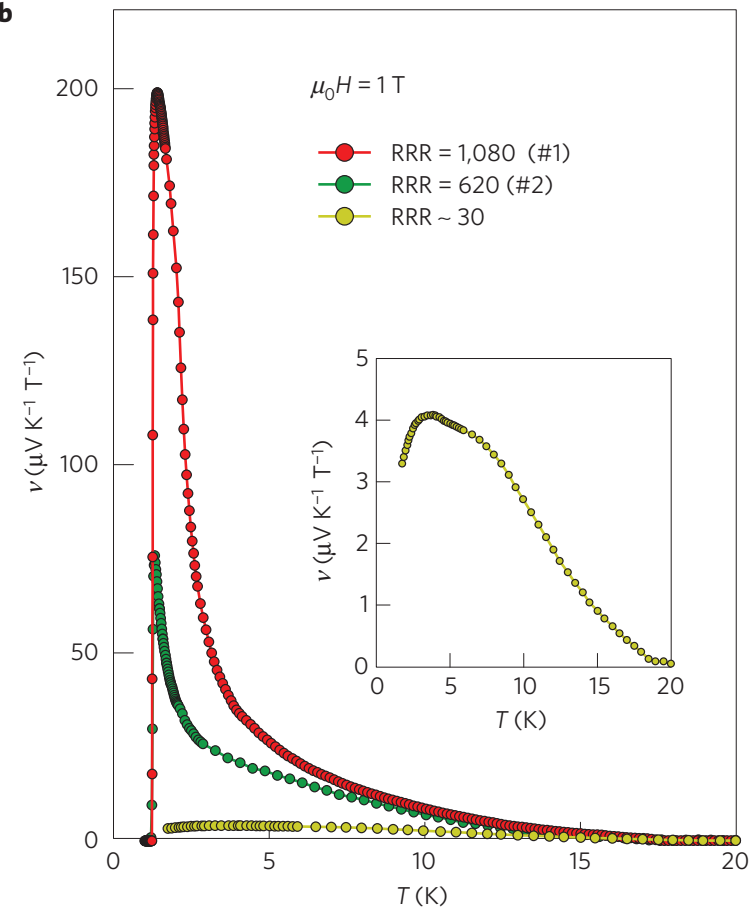

Figure 2 | Anomalously large Nernst signal and thermomagnetic figure of merit. a, The $T$-dependence of $v$ (left scale) and $\rho_{x x}$ (right scale) measured at $\mu_{0} H=1 \mathrm{~T}$ near the superconducting transition. Both $v$ and $\rho_{x x}$ vanish at the vortex lattice melting transition temperature $T_{\text {melt }}$. $\mathbf{b}$, Comparison of the $v(T)$ data at $\mu_{0} H=1 \mathrm{~T}$ between samples with different scattering rates ( $R R R=1,080,620$ and 30). The data for RRR 30 (expanded in the inset) is taken from ref. 23. c, Thermomagnetic figure of merit $Z T_{\epsilon}=N^{2} \sigma T / \kappa$ at $1.5 \mathrm{~K}$ as a function of field in crystal $\# 1$ of $\mathrm{URu}_{2} \mathrm{Si}_{2}$ (red diamonds), compared with previous data in the semimetals $\mathrm{PrFe}_{4} \mathrm{P}_{12}$ (blue line) and Bi (black line) at $1.2 \mathrm{~K}$ taken from ref. 24.

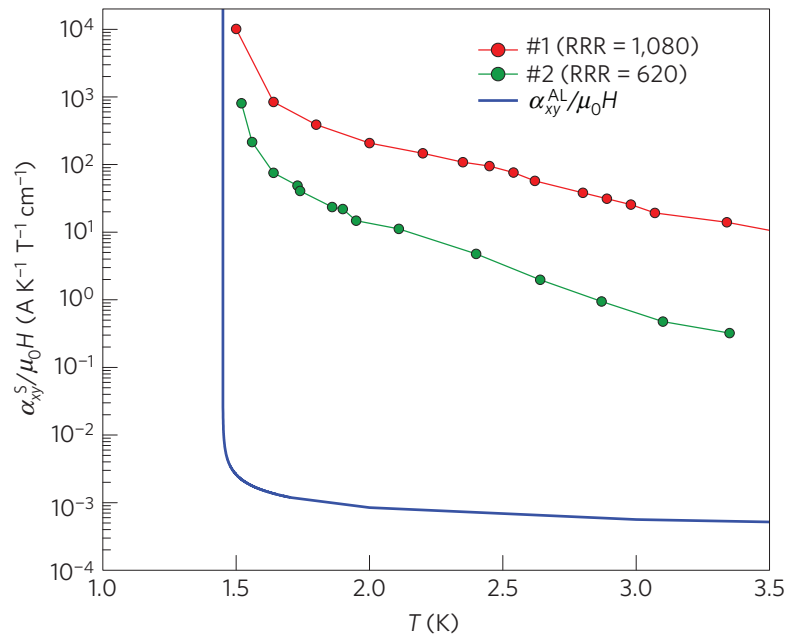

Figure 3 | Comparison with the standard theory of superconducting fluctuations. $T$-dependence of the fluctuation-induced Peltier coefficient divided by the magnetic field, $\alpha_{x y}^{S} / \mu_{0} H$, for crystals \#1 and \#2. The blue line represents the Peltier coefficient that results from Gaussian-type (Aslamazov-Larkin) fluctuations, given by equation (1).

whose effective $H_{c 2}$ corresponds to less than $1 \mathrm{mOe}$, are required. The multiband effect is, therefore, highly unlikely to explain the observed $\alpha_{x y}^{S}$. Third, the characteristic temperature scale of phase fluctuations is given as $T_{\Theta}=A\left(\hbar^{2} a / 4 \mu_{0} k_{\mathrm{B}} \mathrm{e}^{2} \lambda_{a b}^{2}(0)\right)$, where $a=\sqrt{2} \xi_{c}(0), \lambda_{a b}$ is the in-plane penetration depth and $A$ is a

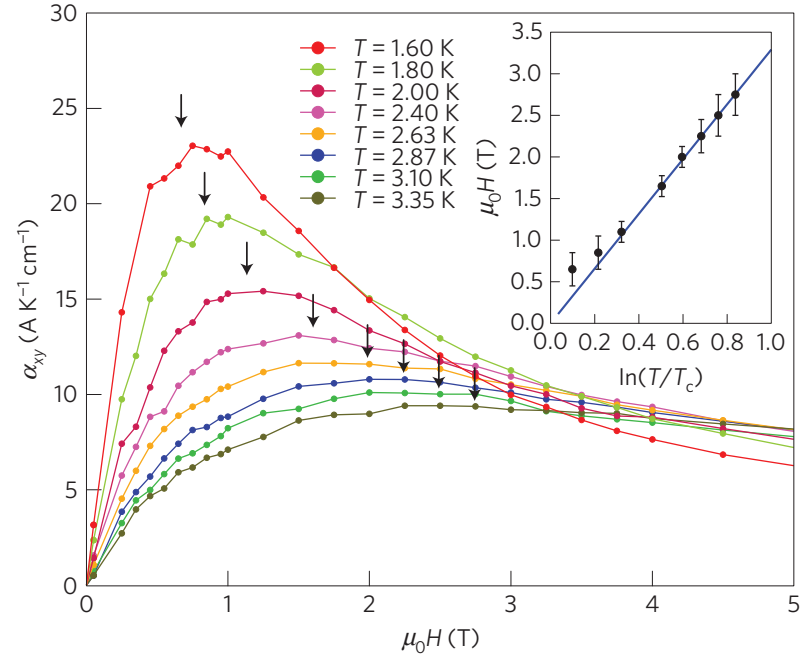

Figure 4 | Superconducting fluctuations and the ghost critical field. $\mathrm{H}$-dependence of the Peltier coefficient $\alpha_{x y}=v \mu_{0} H / \rho_{x x}$ of crystal \#2 at various temperatures. Arrows mark the peak fields. Inset: peak field plotted as a function of $\ln \left(T / T_{\mathrm{C}}\right)$. Error bars are due to the uncertainties in determining the peak positions. The solid line represents $\mu_{0} H^{*}=\Phi_{0} /\left(2 \pi \xi_{a b}^{2}(0)\right) \ln \left(T / T_{c}\right)$, with $\xi_{a b}(0)=10 \mathrm{~nm}$, which is the so-called ghost critical field.

dimensionless number of the order of unity ${ }^{27}$. Using $A=2$ and $\lambda_{a b}=0.8 \mu \mathrm{m}$ (ref. 6), we obtain $T_{\Theta} / T_{c} \sim 100$, suggesting that the phase fluctuations are not important. 
The colossal thermomagnetic response in $\mathrm{URu}_{2} \mathrm{Si}_{2}$ seems to point to a new type of superconducting fluctuation generated by a degree of freedom which has not been hitherto taken into account. It is tempting to consider that such a degree of freedom is intimately related to the superconducting state with broken TRS, because one of the most essential differences between $\mathrm{URu}_{2} \mathrm{Si}_{2}$ and $\mathrm{CeCoIn}$, with no discernible $v^{\mathrm{S}}$, is that TRS is broken in the former whereas it is not broken in the latter ${ }^{28}$. In fact, a chirality-induced anomalous Nernst signal has been pointed out in ref. 29. Very recently, it has been shown that the chirality or Berry-phase associated with the superconducting state with broken TRS gives rise to a new type of fluctuation ${ }^{30}$, showing that $\alpha_{x y}^{S}(T)$ is strikingly enhanced with $\tau$ and its $T$-dependence is different from that predicted by the Gaussian fluctuation theories (Supplementary Information). As shown in Supplementary Fig. 4, the experimental results are reproduced well within the framework of the model of Berry-phase fluctuations. The present results suggest that superconducting fluctuations contain a key ingredient for the topological nature of superconductors, which is a new frontier of condensed matter physics.

\section{Methods}

The ultraclean single crystals of $\mathrm{URu}_{2} \mathrm{Si}_{2}$ were grown by the Czochralski pulling method in a tetra-arc furnace ${ }^{21}$. The well-defined superconducting transition was confirmed by the specific heat measurements. The Nernst and Seebeck coefficients were measured by the standard d.c. method with one resistive heater, two Cernox thermometers and two lateral contacts (lower inset of Fig. 1a). For the accurate determination of these coefficients, the crystals are cut in a rectangular shape, with the long direction corresponding to the $a$ crystalline axis. The dimensions are $1.9 \times 0.56 \times 0.13 \mathrm{~mm}^{3}(\# 1)$ and $2.0 \times 0.60 \times 0.15 \mathrm{~mm}^{3}(\# 2)$. Magnetic field is applied perpendicular to the cleavage $a b$-plane with a misalignment less than $1^{\circ}$.

Received 16 May 2014; accepted 30 October 2014; published online 1 December 2014

\section{References}

1. Larkin, A. I. \& Varlamov, A. A. Theory of Fluctuations in Superconductors (Oxford Univ. Press, 2005).

2. $\mathrm{Xu}, \mathrm{Z}$. A. et al. Vortex-like excitations and the onset of superconducting phase fluctuation in underdoped $\mathrm{La}_{2-x} \mathrm{Sr}_{x} \mathrm{CuO}_{4}$. Nature 406, 486-488 (2000).

3. Wang, Y., Li, L. \& Ong, N. P. Nernst effect in high- $T_{c}$ superconductors. Phys. Rev. B 73, 024510 (2006).

4. Kasahara, Y. et al. Exotic superconducting properties in the electron-hole-compensated heavy-fermion semimetal $\mathrm{URu}_{2} \mathrm{Si}_{2}$. Phys. Rev. Lett. 99, 116402 (2007).

5. Yano, K. et al. Field-angle-dependent specific heat measurements and gap determination of a heavy fermion superconductor $\mathrm{URu}_{2} \mathrm{Si}_{2}$. Phys. Rev. Lett. 100, 017004 (2008).

6. Okazaki, R. et al. Anomalous temperature dependence of lower critical field in ultraclean $\mathrm{URu}_{2} \mathrm{Si}_{2}$. J. Phys. Soc. Jpn 79, 084705 (2010).

7. Li, G. et al. Bulk evidence for a time reversal symmetry broken superconducting state in $\mathrm{URu}_{2} \mathrm{Si}_{2}$. Phy. Rev. B 88, 134517 (2013).

8. Rullier-Albenque, F. et al. Nernst effect and disorder in the normal state of high- $T_{\mathrm{c}}$ cuprates. Phys. Rev. Lett. 96, 067002 (2006).

9. Pourret, A. et al. Observation of the Nernst signal generated by fluctuating Cooper pairs. Nature Phys. 2, 683-686 (2006).

10. Li, P. \& Greene, R. L. Normal-state Nernst effect in electron-doped $\mathrm{Pr}_{2-x} \mathrm{Ce}_{x} \mathrm{CuO}_{4-\delta}$ : Superconducting fluctuations and two-band transport. Phys. Rev. B 76, 174512 (2007).

11. Pourret, A., Spathis, P., Aubin, H. \& Behnia, K. Nernst effect as a probe of superconducting fluctuations in disordered thin films. New J. Phys. 11, 055071 (2009).
12. Chang, J. et al. Decrease of upper critical field with underdoping in cuprate superconductors. Nature Phys. 8, 751-756 (2012).

13. Ussishkin, I., Sondhi, S. L. \& Huse, D. A. Gaussian superconducting fluctuations, thermal transport, and the Nernst effect. Phys. Rev. Lett. 89, 287001 (2002).

14. Oganesyan, V. \& Ussishkin, I. Nernst effect, quasiparticles, and $d$-density waves in cuprates. Phys. Rev. B 70, 054503 (2004).

15. Podolsky, D., Raghu, S. \& Vishwanath, A. Nernst effect and diamagnetism in phase-fluctuating superconductors. Phys. Rev. Lett. 99, 117004 (2007).

16. Serbyn, M. N., Skvortsov, M. A., Varlamov, A. A. \& Galitski, V. Giant Nernst effect due to fluctuating Cooper pairs in superconductors. Phys. Rev. Lett. 102, 067001 (2009).

17. Michaeli, K. \& Finkel'stein, A. M. Fluctuations of the superconducting order parameter as an origin of the Nernst effect. Europhys. Lett. 86, 27007 (2009).

18. Levchenko, A., Norman, M. R. \& Varlamov, A. A. Nernst effect from fluctuating pairs in the pseudogap phase of the cuprates. Phys. Rev. B 83, 020506 (2011).

19. Mydosh, J. A. \& Oppeneer, P. M. Colloquium: Hidden order, superconductivity, and magnetism: The unsolved case of $\mathrm{URu}_{2} \mathrm{Si}_{2}$. Rev. Mod. Phys. 83, 1301-1322 (2011).

20. Goswami, P. \& Balicas, L. Topological properties of possible Weyl superconducting states of $\mathrm{URu}_{2} \mathrm{Si}_{2}$. Preprint at http://arxiv.org/abs/ 1312.3632 (2013)

21. Matsuda, T. D. et al. Details of sample dependence and transport properties of $\mathrm{URu}_{2} \mathrm{Si}_{2}$. J. Phys. Soc. Jpn 80, 114710 (2011).

22. Behnia, K. The Nernst effect and the boundaries of the Fermi liquid picture. J. Phys. Condens. Matter 21, 113101 (2009).

23. Bel, R. et al. Themoelectricity of $\mathrm{URu}_{2} \mathrm{Si}_{2}$ : Giant Nernst effect in the hidden-order state. Phys. Rev. B 70, 220501 (2004).

24. Behnia, K., Méasson, M-A. \& Kopelevich, Y. Nernst effect in semimetals: The effective mass and the figure of merit. Phys. Rev. Lett. 98, 076603 (2007).

25. Bel, R. et al. Giant Nernst effect in CeCoIn ${ }_{5}$. Phys. Rev. Lett. 92, 217002 (2004).

26. Izawa, K. et al. Thermoelectric response near a quantum critical point: The case

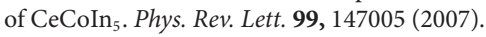

27. Emery, V. J. \& Kivelson, S. A. Importance of phase fluctuations in superconductors with small superfluid density. Nature 374, 434-437 (1995).

28. Higemoto, W. et al. $\mu$ SR studies on heavy fermion superconductors CeIrIn and CeCoIn ${ }_{5}$. J. Phys. Soc. Jpn 71, 1023-1026 (2002).

29. Kotetes, P. \& Varelogiannis, D. Chirality induced tilted-hill giant Nernst signal. Phys. Rev. Lett. 104, 106404 (2010).

30. Sumiyoshi, H. \& Fujimoto, S. Giant Nernst and Hall effects in chiral superconductors due to Berry phase fluctuations. Preprint at http://arxiv.org/abs/1403.3977 (2014).

\section{Acknowledgements}

We thank K. Behnia, R. Ikeda and A. Kapitulnik for fruitful discussions, and S. Tonegawa for technical assistance. This work was supported by Grants-in-Aid for Scientific Research (KAKENHI) from Japan Society for the Promotion of Science (JSPS), and by the 'Topological Quantum Phenomena' (No. 25103713) Grant-in-Aid for Scientific Research on Innovative Areas from the Ministry of Education, Culture, Sports, Science and Technology (MEXT) of Japan. This work at MSU (A.L.) was supported by NSF Grant No. DMR-1401908.

\section{Author contributions}

Y.H., T.D.M., E.Y. and Y.O. prepared the samples. T.Y. and Y.S. carried out the measurements. T.Y., Y.S., H.S., S.F., A.L., T.S. and Y.M. interpreted and analysed the data. A.L., T.S. and Y.M. wrote the manuscript.

\section{Additional information}

Supplementary information is available in the online version of the paper. Reprints and permissions information is available online at www.nature.com/reprints. Correspondence and requests for materials should be addressed to Y.M.

\section{Competing financial interests}

The authors declare no competing financial interests. 